\title{
Tailored sPP/Silica Nanocomposite for Ecofriendly Insulation of Extruded HVDC Cable
}

\author{
Bin Dang, Jinliang He, Jun Hu, and Yao Zhou \\ State Key Laboratory of Power Systems, Department of Electrical Engineering, Tsinghua University, Beijing 100084, China \\ Correspondence should be addressed to Jinliang He; hejl@tsinghua.edu.cn
}

Received 7 September 2015; Revised 14 November 2015; Accepted 19 November 2015

Academic Editor: Ilaria Armentano

Copyright ( 2015 Bin Dang et al. This is an open access article distributed under the Creative Commons Attribution License, which permits unrestricted use, distribution, and reproduction in any medium, provided the original work is properly cited.

\begin{abstract}
Cross-linked polyethylene (XLPE) is a thermosetting material that cannot be recycled at the end of its lifetime. This study investigated the potential of syndiotactic polypropylene (sPP)/silica as an ecofriendly extruded insulation system for HVDC cables. We investigated the morphology, Fourier transform infrared, and thermal, thermomechanical, and electrical behaviors of sPP modified with $0.5-3 \%$ nanosilica. We found that the silica/sPP nanocomposite without cross-linking offered a suitable mechanical modulus at room temperature and sufficient intensity at high temperatures, and adding nanosilica modified by a silane coupling agent to the sPP resulted in significant DC resistivity and space charge improvement. The optimal nanosilica content in the sPP was determined by balancing the mechanical and thermomechanical characteristics and the DC resistivity. The sPP/silica nanocomposite reported here shows great potential as a candidate insulation material for future ecofriendly extruded HVDC cables.
\end{abstract}

\section{Introduction}

Extruded cables with cross-linked polyethylene (XLPE) insulation have been successfully applied for high-voltage direct current transmission in power systems because of their favorable combination of superior mechanical, thermomechanical, and electrical properties. However, it is difficult to recycle the XLPE when the cable reaches the end of its life, because of the thermosetting properties of XPLE; it must therefore be burnt, resulting in great damage to the environment. Minimizing the detrimental environmental effects that result from the disposal of XLPE is important. The difficulty in producing recyclable power cable insulation lies in combining good mechanical properties at room temperature with good thermomechanical properties at the operating temperature.

Recent studies have investigated novel thermoplastic alternatives for the electrical insulation of extruded cables [15]. The potential replacements for XLPE for next-generation extruded cables can be divided into ethylene and propylene systems. For ethylene systems, low-density polyethylene (LDPE) and its blends [1], high-density polyethylene (HDPE) and its blends [2], and LDPE/HDPE blends [3] have been investigated. It was found that LDPE and HDPE have a lower operating temperature than polypropylene and that HDPE is too rigid to be used with cables and must be blended with elastomer. Another candidate material for cables is polypropylene; polypropylene has a higher melting temperature than polyethylene. However, a series of experiments on isotactic polypropylene (iPP) showed that iPP was a stiff material that lacked the requisite flexibility at room temperature that would allow it to be incorporated into cables. Researchers have studied propylene-based copolymers [4] and alloys [5]. Unfortunately, studies [5] revealed that none of the iPP-based systems satisfied all of the requirements of an ideal thermoplastic insulation system, namely, lowtemperature flexibility, high-temperature integrity, and high breakdown strength.

Syndiotactic polypropylene (sPP), which has a potentially sufficient flexibility, in contrast with iPP, and low dielectric loss and high breakdown strength [6], is a promising candidate for new insulation materials. The disadvantage of sPP is its slow crystallization rate, which will limit its commercial application [7]. The addition of nanoparticles can enhance the crystallization rate, thus reducing the processing time. Polymer nanocomposites have attracted much attention for 
TABLE 1: Materials used in this investigation.

\begin{tabular}{lcc}
\hline Abbreviation & \multicolumn{2}{c}{ Composition (weight ratio) } \\
& PP & chl-silica \\
\hline sPP & 100 & 0 \\
$0.5 \mathrm{phr}$ & 100 & 0.5 \\
$1 \mathrm{phr}$ & 100 & 1 \\
$3 \mathrm{phr}$ & 100 & 3 \\
\hline
\end{tabular}

the insulation of HVDC, based on polyethylene copolymers, because of their improved electrical properties [7]. Nanoparticles have a larger interfacial area than microcomposites and can thus determine the properties of nanofillers, even at low volume fractions in such fillers [8]. However, the nanoparticles have a strong tendency to aggregate, which may degrade the performance of the nanocomposites. Such nanoparticles are therefore typically functionalized, to avoid agglomeration and maintain the stability of the nanoparticles within the polymer matrix [9].

In this study, by analyzing the morphology, Fourier transform infrared spectra, thermal and thermomechanical characteristics, tensile testing, DC resistivity, and space charge characteristics of sPP/silica nanocomposites, the most suitable candidate materials to meet the ever-growing demand for ecofriendly, highly efficient HVDC cables were identified.

\section{Experiments}

2.1. Materials. Commercial syndiotactic polypropylene 1471 (Total Fina, France) with a melt flow index of $4 \mathrm{~g} / 10 \mathrm{~min}$, a density of $0.88 \mathrm{~g} / \mathrm{cm}^{3}$, and a melting point of $130^{\circ} \mathrm{C}$ was used. Syndiotactic polypropylene 1471 is a low-crystallinity, syndiotactic form of copolymer polypropylene. Nanosilica (SigmaAldrich, USA) with an average diameter of approximately 5$15 \mathrm{~nm}$ was used as the nanoparticles. Dimethyldichlorosilane (hereafter chl) (Sinopharm Chemical Reagent Beijing Co., Ltd., China) was used as a surface modification agent.

2.2. Functionalization of the Silica Nanoparticles. The surface modification of the original silica nanoparticles was performed as follows [10]: The silica was vacuum dried for $24 \mathrm{~h}$ immediately prior to compounding. Then, $10 \mathrm{~g}$ of silica and $100 \mathrm{~mL}$ of xylene were mixed in a beaker. After sonication for $30 \mathrm{~min}$, the mixture was transferred to a three-necked, roundbottom flask, and $5 \mathrm{~g}$ chl was added to the flask. This mixture was heated to $130^{\circ} \mathrm{C}$ in an oil bath and refluxed for $12 \mathrm{~h}$ under magnetic stirring. The nanoparticles were washed with deionized water and separated from the reaction mixture via centrifugation performed at $4000 \mathrm{rpm}$ for $30 \mathrm{~min}$; they were then dried under vacuum at $100^{\circ} \mathrm{C}$ for $24 \mathrm{~h}$.

2.3. Preparation of sPP/Silica Nanocomposite Films. Four propylene-based systems were studied, as shown in Table 1. The systems were manually premixed, and the mixture was melt blended in a HAKKE minilab II at $200^{\circ} \mathrm{C}$ for $5 \mathrm{~min}$. All samples were manufactured using hot pressing. $150 \mu \mathrm{m}$ films were prepared via premelting performed using a curing press operated at $200^{\circ} \mathrm{C}$ for $10 \mathrm{~min}$, followed by pressing under a pressure of $\sim 20 \mathrm{MPa}$ for $10 \mathrm{~min}$ and pressing at $25^{\circ} \mathrm{C}$ under a pressure of $\sim 5 \mathrm{MPa}$ for a further $10 \mathrm{~min}$.

All samples were sandwiched between two pieces of cover steel plate under a short-circuit state and annealed at $100^{\circ} \mathrm{C}$ for $2 \mathrm{~h}$ in the vacuum chamber, to estimate the residual stress.

2.4. Characterization. To investigate the dispersibility of the chl-silica nanoparticles in the sPP matrix and the morphology, cross-sectional scanning electron microscopy (SEM) images were obtained using an ultrahigh-resolution HITACHI S-5500 instrument operated at $5 \mathrm{kV}$. The samples were fractured in liquid nitrogen and then coated with carbon prior to examination under the electron beam. Fourier transform infrared (FTIR) spectra were measured over the range of $4000-500 \mathrm{~cm}^{-1}$, using a Nicolet 6700 FTIR instrument. Differential scanning calorimetry (DSC) measurements on $\sim 5 \mathrm{mg}$ samples were performed under a nitrogen atmosphere, using a heating/cooling rate of $10^{\circ} \mathrm{C} \mathrm{min}^{-1}$ between $20^{\circ} \mathrm{C}$ and $150^{\circ} \mathrm{C}$, using a TA Q2000 instrument. The thermal stability of $\sim 10 \mathrm{mg}$ samples was determined using a TGA Q5000 series thermogravimetric analyzer (TA Instruments, USA), using a heating rate of $10^{\circ} \mathrm{C} \mathrm{min}^{-1}$ in an ambient air environment. Dynamic mechanical thermal analysis (DMTA) was performed using a TA Q800 instrument. The investigated samples (with dimensions of $20 \mathrm{~mm} \times 5 \mathrm{~mm}$ ) were cooled to $-30^{\circ} \mathrm{C}$ and heated to $120^{\circ} \mathrm{C}$ under nitrogen flow, using a heating rate of $3^{\circ} \mathrm{C} \mathrm{min}^{-1}$, under a strain-controlled sinusoidal tensile loaded at a frequency of $1 \mathrm{~Hz}$ and a strain of $0.01 \%$. Tensile testing was undertaken at different temperature using a TA Q800 instrument at a fixed speed of $250 \mu \mathrm{m} \mathrm{min}{ }^{-1}$. Rectangle specimens with a cross section of $5 \mathrm{~mm} \times 2 \mathrm{~mm}$ were prepared for the measurements. DC resistivity measurements were performed using a three-terminal-cell system, according to ASTM D257-14. The applied DC electrical field was $6 \mathrm{kV} \mathrm{mm}^{-1}$, and the measurements were performed for $60 \mathrm{~min}$, long enough for the polarization current to reach a steady state. The DC electrical field of $60 \mathrm{kV} / \mathrm{mm}$ was applied to the plate samples for 1800 seconds, using an electric pulse with an amplitude of $800 \mathrm{~V}$ and duration of $7 \mathrm{~ns}$. The space charge measurements were carried out using a pulsed electroacoustic method (PEA) system equipped with a Lecroy WaveRunner 610Zi (1 GHz, $20 \mathrm{GS} /$ s digital oscilloscope).

\section{Results and Discussion}

3.1. FTIR Characterization. The surface modification of inorganic nanoparticles enlarges the interface between the matrix and the nanoparticles, leading to improvements in the performance of the nanocomposites. The silica nanoparticles, which were functionalized via surface modification, were characterized using FTIR, as shown in Figure 1. The silica, which had $\mathrm{O}-\mathrm{H}$ groups, showed an absorption peak at $3427 \mathrm{~cm}^{-1}$ [16]. The FTIR spectra clearly illustrated the presence of fewer silicon hydroxyls in the chl-silica, indicating the enhancement of the surface hydrophobicity of the silica nanoparticles after the surface modification. Another change in the FTIR spectra was related to the wavenumbers at $2966 \mathrm{~cm}^{-1}$ and $2870 \mathrm{~cm}^{-1}$, 


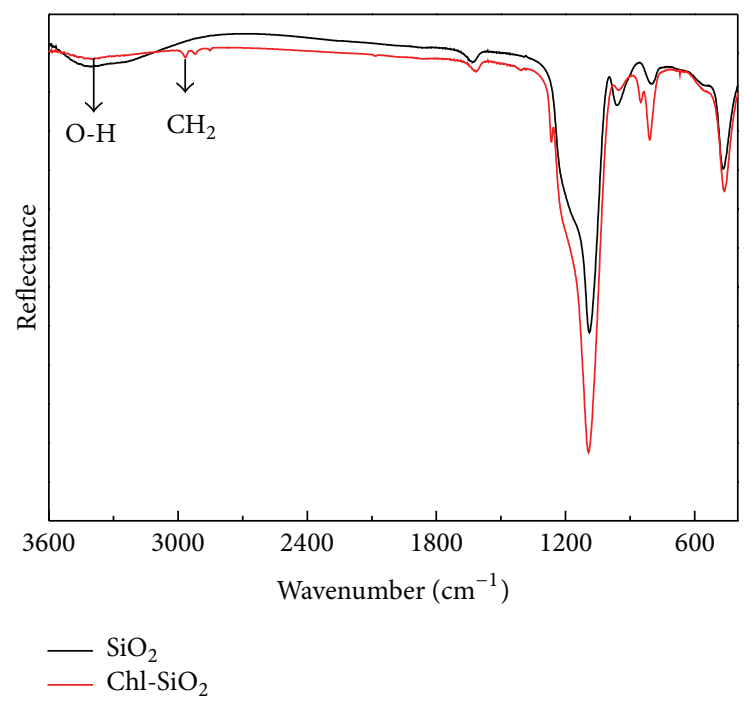

FIGURE 1: FTIR spectra for silica nanoparticles.

which represented carbon hydrogen bonds. Compared with the lack of absorption at these two wavenumbers for the silica nanoparticles, the presence of absorption signals for the chl coupling agent indicated that the chl had been successfully grafted to the surface of the silica nanoparticles.

3.2. Dispersion and Morphology. Particle dispersion is the key factor in controlling the properties of nanocomposites. Figure 2 shows SEM images illustrating the dispersion of the nanoparticles in the nanocomposites with $3 \mathrm{phr}$ chlsilica. Similar images were obtained for the other samples. The images suggested that the silica nanoparticles with a polymer shell were well dispersed in the sPP matrix, with good compatibility. It is likely that the excellent compatibility of the silica nanoparticles and the SPP matrix originated from the surface modification with the silane coupling agent.

3.3. Thermal Analysis. Figure 3 presents the melting and crystallization behaviors of the sPP/chl-silica nanocomposites as a function of the chl-silica content. Figure 3(a) shows that the sPP nanocomposites had two distinct melting transitions, in accordance with results from a previous study [17]. The melting temperature of the neat $\mathrm{SP}$ was higher than that of LDPE or LLDPE, and that melting temperature shifted to a higher temperature when the chl-silica was incorporated into the sPP matrix. The sPP nanocomposites therefore all appear to be good candidates for HVDC cable applications, at least from the point of view of their melting behavior.

The nonisothermal crystallization curves for the pure sPP and its nanocomposites shown in Figure 3(b) indicate that the crystallization temperature moved to a higher temperature when the chl-silica was added to the sPP matrix, proving that incorporating the chl-silica had a great effect on the nucleation of the nanocomposites. The higher the crystallization temperature, the shorter the cooling time. Hence, the sPP nanocomposites had a shorter processing cycle compared with sPP.
TABLE 2: Degradation temperatures for the sPP/chl-silica samples, obtained from TGA results.

\begin{tabular}{lc}
\hline Sample & $T_{\text {degrad }}\left({ }^{\circ} \mathrm{C}\right)$ \\
\hline sPP & 333.5 \\
$0.5 \mathrm{phr}$ & 331.5 \\
$1 \mathrm{phr}$ & 347.6 \\
$3 \mathrm{phr}$ & 352.8 \\
\hline
\end{tabular}

Thermogravimetric analysis was performed in an attempt to understand the degradation characteristics of the samples, which are important for the processing of the power cable. The thermogravimetry (TG) and derivative thermogravimetry (DTG) results are shown in Figure 4. The temperatures at which the maximum rate of heat consumption occurred for this degradation process are listed in Table 2 for the various nanocomposites. Table 2 shows that sPP with $1 \mathrm{phr}$ and $3 \mathrm{phr}$ chl-silica had a maximum decomposition temperature of $347.6^{\circ} \mathrm{C}$ and $352.8^{\circ} \mathrm{C}$, respectively.

3.4. Dynamic Mechanical and Thermal Characteristics. HVDC cables are typically installed at room temperature and operate at high temperatures. It is therefore necessary to focus on the mechanical properties at room temperature and at high temperatures. Figure 5(a) shows a plot of the storage modulus as a function of temperature. The results indicated that all samples had a storage modulus of $\sim 500 \mathrm{MPa}$ at room temperature, lower than that of isotactic polypropylene $(\sim 1000 \mathrm{MPa})$. All of the samples showed a storage modulus of $\sim 100 \mathrm{MPa}$ at high temperatures, higher than that of LDPE or LLDPE.

We determined from the $\tan \delta$ curves in Figure 5(b) that the characteristic peak for polypropylene was at $\sim 12^{\circ} \mathrm{C}$. The peak in the $\tan \delta$ curve was due to the glass transition characteristic of sPP [18]. The presence of the nanosilica did not change the glass transition temperature of the composites.

3.5. Tensile Testing. Figure 6 shows the typical tensile stressstrain curves of sPP nanocomposites at different temperatures. Furthermore, elastic modulus and tensile yield strength of the samples at different temperatures are presented in Figure 7. It can be seen from Figure 6 that all four materials at different temperature displayed a characteristic necking behavior after imposed strain and had high breaking elongation (more than 200\%). The high breaking elongation at room temperature indicates that the nanocomposites have sufficiently high mechanical flexibility for cable installation. From Figure 7, the elastic modulus increased and the tensile yield strength decreased at different temperatures when the chl-silica was added to the sPP matrix. In addition, an elastic modulus at room temperature of $\sim 110 \mathrm{MPa}$ and sufficient elastic modulus at high temperature for sPP nanocomposites indicate that the nanocomposites are suitable for cable applications [11].

3.6. DC Resistivity. The dielectric loss from HVDC cables results mainly from the leakage current. Figure 8 shows the 


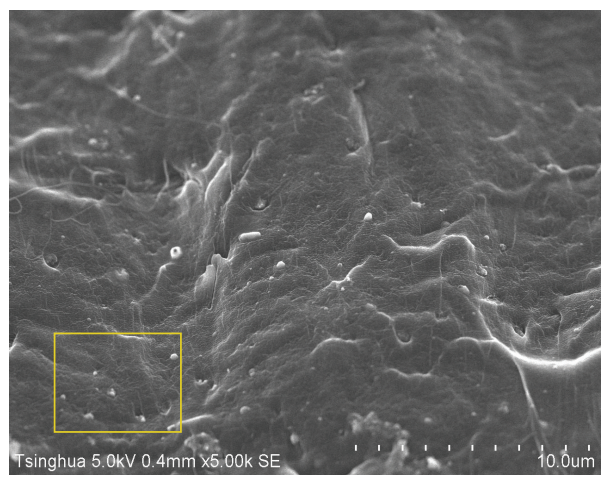

(a)

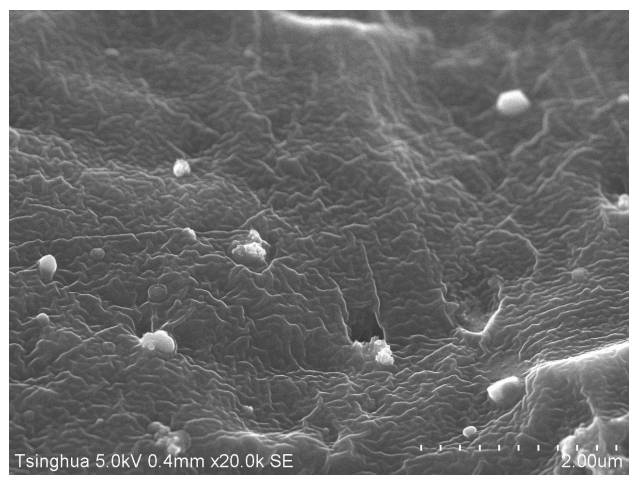

(b)

FIGURE 2: SEM images demonstrating the dispersibility of the silica nanoparticles and showing the cross-sectional microstructure of the sample; (b) is a partial enlargement of the frame shown in (a).

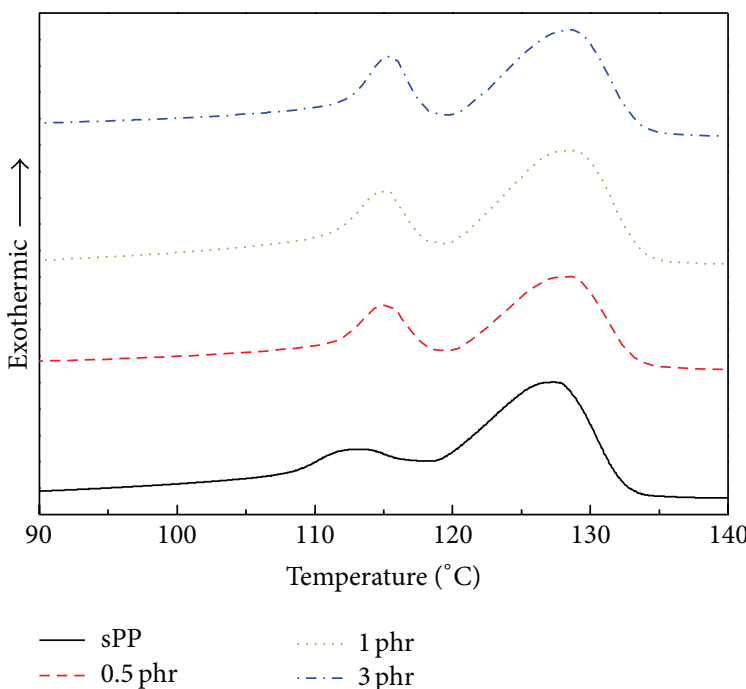

(a)

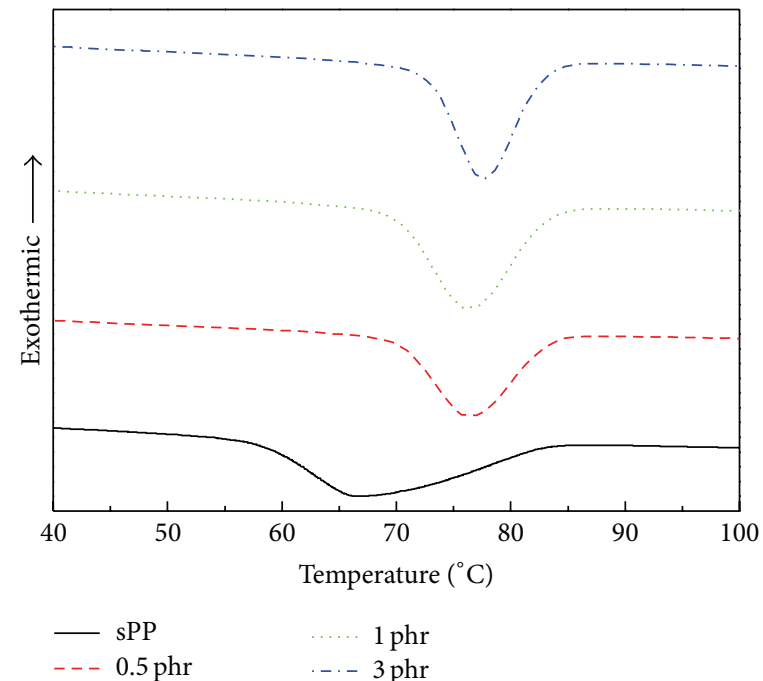

(b)

FIGURE 3: Melting (a) and crystallization (b) curves for the sPP/silica nanocomposites.

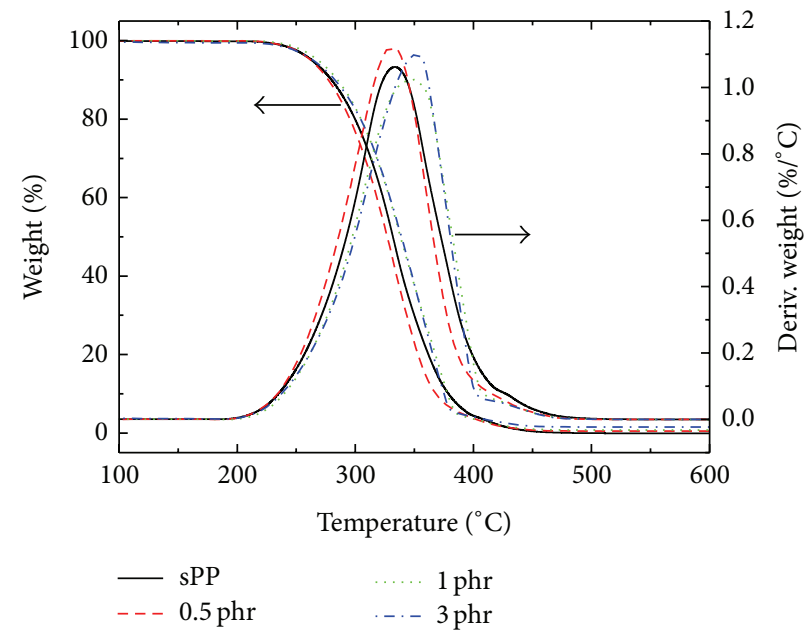

FIGURE 4: TG and DTG curves for the sPP/chl-silica nanocomposites. 


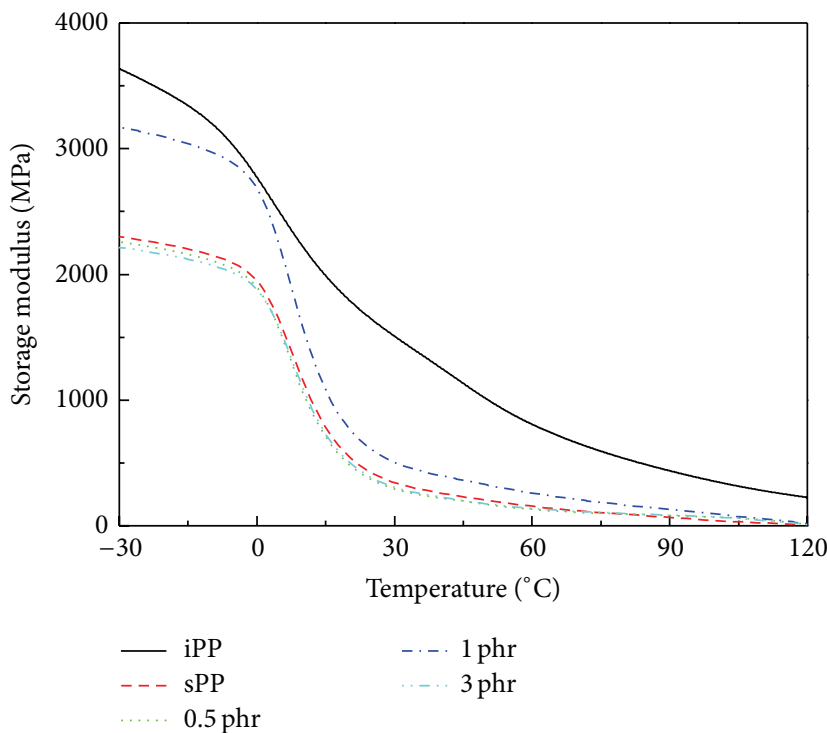

(a)

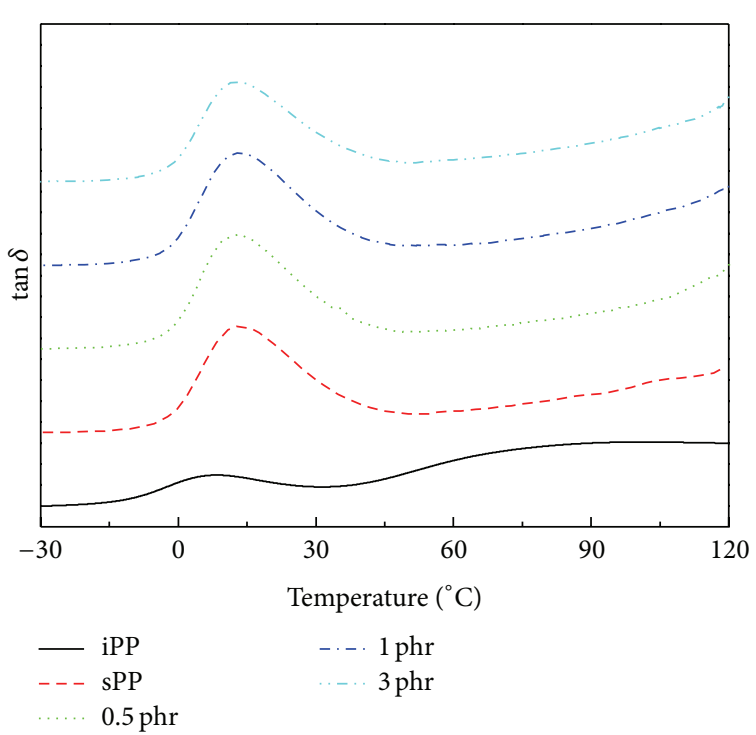

(b)

Figure 5: Storage modulus (a) and $\tan \delta$ (b) for the sPP/chl-silica nanocomposites.

leakage current density and DC resistivity of the samples at room temperature. The pure sPP showed a higher leakage current and took less time to reach stability, compared with the other samples. The electric field distribution within the insulation of an HVDC cable depends on the DC resistivity. The volume resistivity was evaluated from the leakage current value noted 60 minutes after the application of the DC voltage shown in Figure 8(b). Under the applied field, the volume resistivity of the films with silica nanofillers was higher than that of the pure sPP; in particular, the sPP with $1 \%$ chlsilica exhibited a volume resistivity that was one order of magnitude higher than that shown by the base polymer. A possible reason for this was the good dispersion of the chl-silica in the sPP matrix. For polymer nanocomposites, the nanoparticle-polymer interactions play an important role in determining the materials' performance [19]. More specifically, this phenomenon was related to the introduction of deeper carrier traps by the incorporation of the nanosilica [20]. The nanocomposite with $1 \mathrm{phr}$ chl-silica had the lowest leakage current density, showing that this sample had more deep traps than the other samples. These deep traps likely had a significant effect on the space charge distribution in the samples. This effect will be discussed later.

3.7. Space Charge Characteristics. Apart from the mechanical properties and the DC resistivity, an outstanding space charge distribution is another important requirement for HVDC cables, because the accumulation of space charge in HVDC cables results in the failure of cable insulation [13, 21]. Figure 9 shows the space charge distribution in the samples during polarization, under a DC electric field of $60 \mathrm{kV} \mathrm{mm}^{-1}$ applied for $1800 \mathrm{~s}$. Figures 9(a) and 9(d) show that a large number of homocharges formed around the cathode of the neat sPP and the nanocomposite with $3 \mathrm{phr}$ chl-silica; this was a result of electron injection at the cathode. Figures $9(\mathrm{~b})$ and $9(\mathrm{c})$ illustrate the improved space charge distribution in the nanocomposites with $0.5 \mathrm{phr}$ and $1 \mathrm{phr}$ chl-silica. Only a small number of homospace charges were observed near the cathode of these samples. The suppression of space charges is considered to be closely related to the presence of carrier traps. The deep traps in the sPP samples with $0.5 \mathrm{phr}$ or $1 \mathrm{phr}$ chl-silica blocked the electrons injected from the cathode, and the blocked electrons increased the potential barrier for charge injection, thus suppressing the formation of space charges to a significant degree. The sPP with $3 \mathrm{phr}$ chl-silica showed fewer deep traps than the sPP with $0.5 \mathrm{phr}$ chlsilica or that with $1 \mathrm{phr}$ chl-silica, as shown by the leakage current density curves in Figure 8(a). Hence, a small number of electrons could still be injected into the sample with $3 \mathrm{phr}$ chl-silica.

3.8. Comparison between sPP Nanocomposites and XLPE. In order to better illustrate the properties of sPP nanocomposites, a comparison of the various performances considered in this investigation between sPP nanocomposites and typical XLPE is given in Table 3 . The operating temperature of $1 \mathrm{phr}$ is the same as XLPE from the view of thermal and mechanical properties. The electrical properties of $1 \mathrm{phr}$ are more superior to XLPE (i.e., increased volume resistivity and no space charge accumulation).

\section{Conclusion}

In this study, the properties of nanosilica-modified sPP were investigated, and the main conclusions are summarized below:

(1) The silica nanoparticles used for the modification showed excellent dispersion, because of the improvement of the hydrophobicity of silica nanoparticles that 


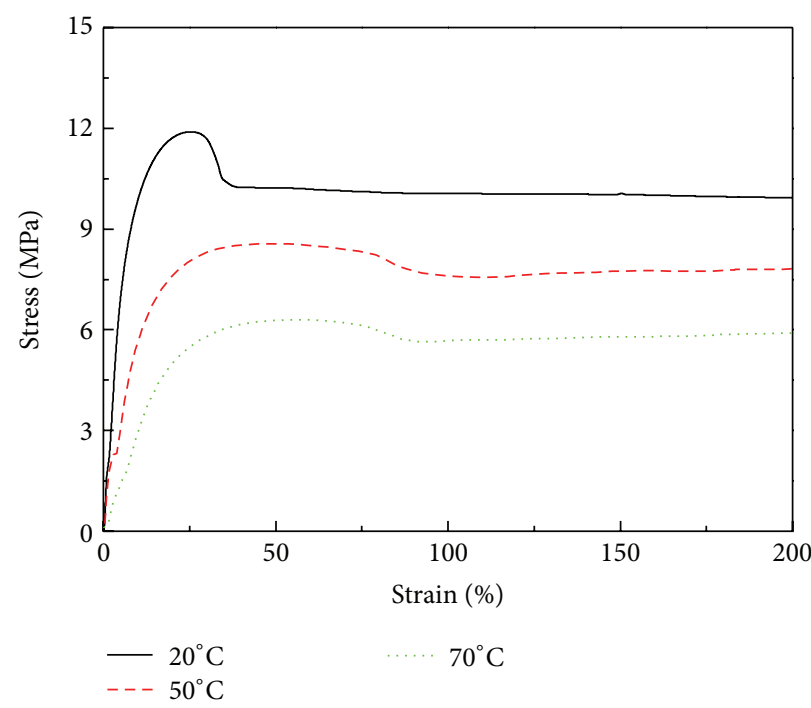

(a)

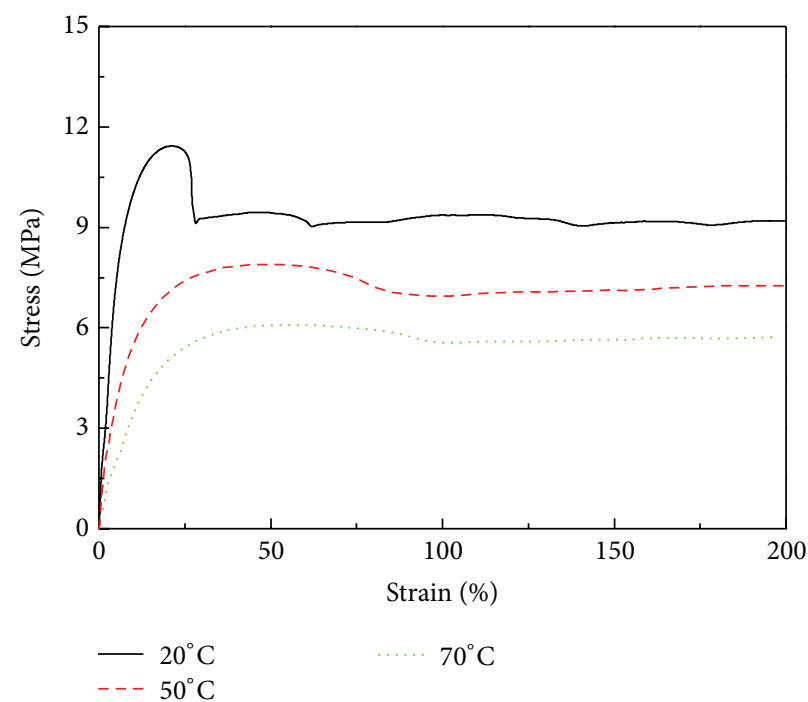

(c)

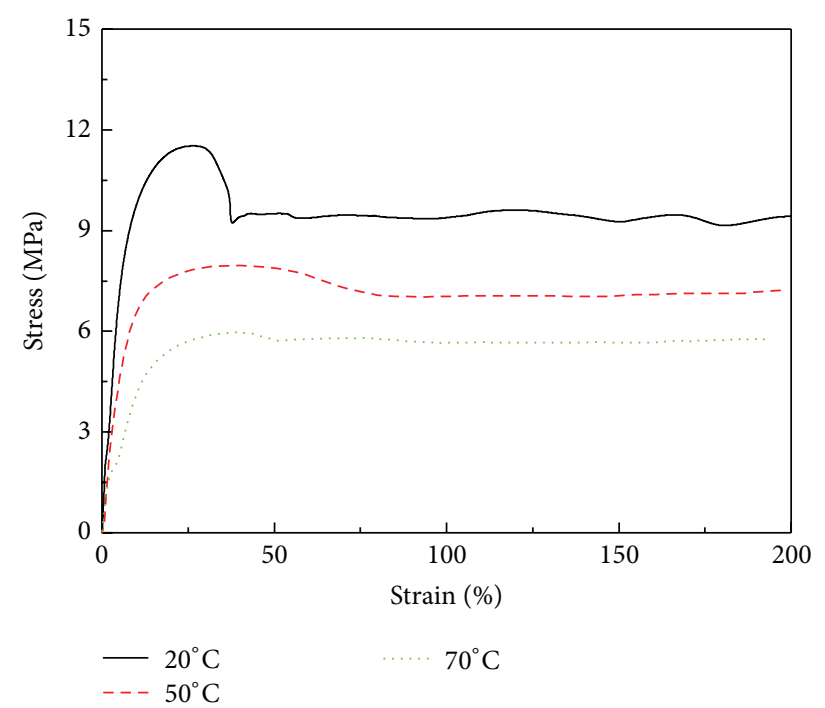

(b)

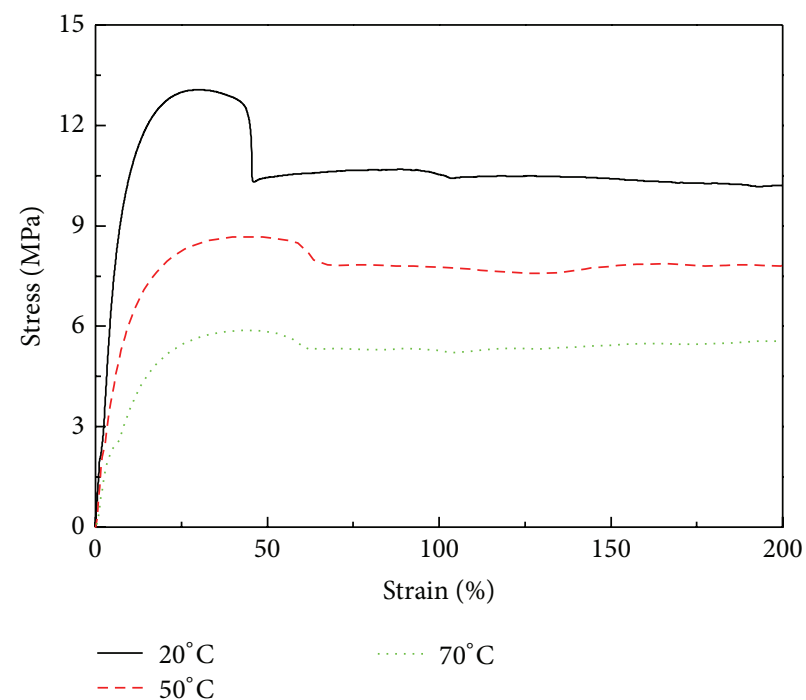

(d)

Figure 6: Tensile tests results at different temperatures (a) sPP, (b) $0.5 \mathrm{phr}$, (c) $1 \mathrm{phr}$, and (d) 3 phr.

resulted from their functionalization, as characterized using FTIR spectroscopy.

(2) The materials without cross-linking provided a suitable mechanical modulus at room temperature and sufficient intensity at high temperatures. The manufactured materials therefore had a sufficient ability to maintain their integrity in a cable under operating conditions.

(3) The melting temperature and crystallization temperature of the $\mathrm{sPP} / \mathrm{chl}$-silica nanocomposites were higher, compared with the values for neat polypropylene.

(4) The addition of chl-silica to the sPP resulted in significant improvement of the DC resistivity. The enhancement in the DC resistivity was related to the introduction of the deep carrier traps by the nanosilica.

Out of the nanocomposites considered here, the nanocomposite containing $1 \%$ nanosilica in sPP provided the optimum mechanical and thermomechanical properties and significant improvements in the DC resistivity and space charge distribution. This nanocomposite holds potential as a candidate insulation material for future ecofriendly extruded HVDC cables.

\section{Conflict of Interests}

The authors declare that there is no conflict of interests regarding the publication of this paper. 


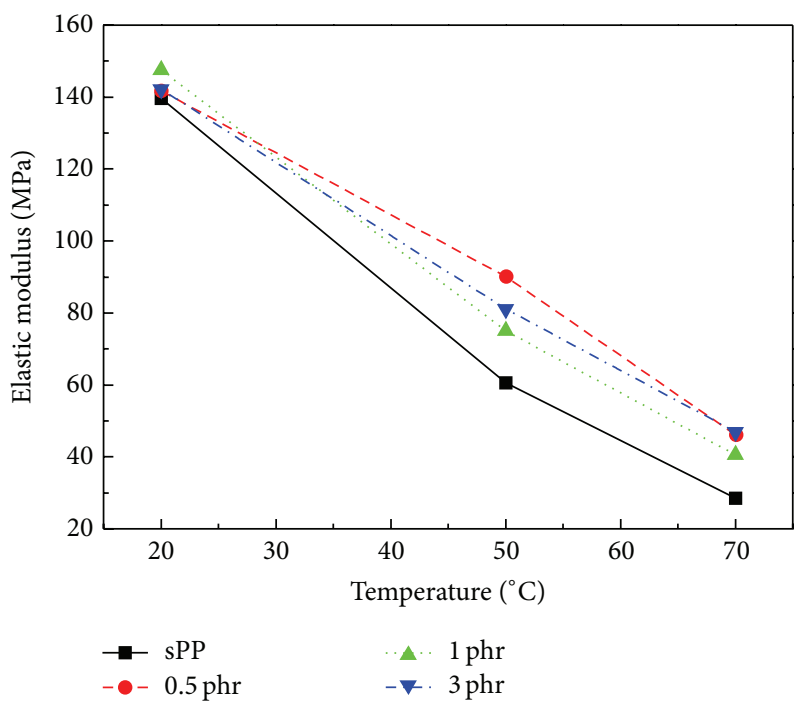

(a)

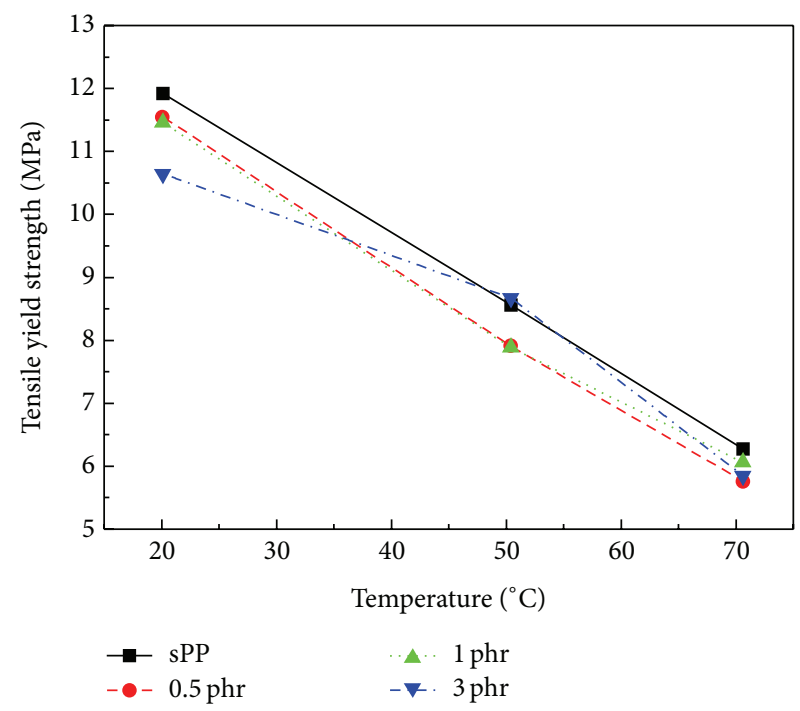

(b)

Figure 7: Elastic modulus (a) and tensile yield strength (b) for sPP nanocomposites at different temperatures.

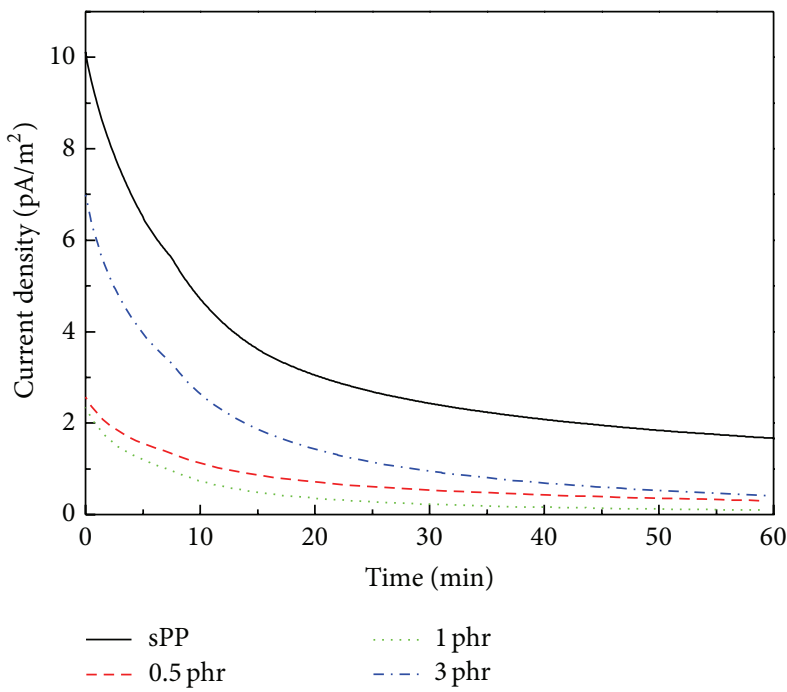

(a)

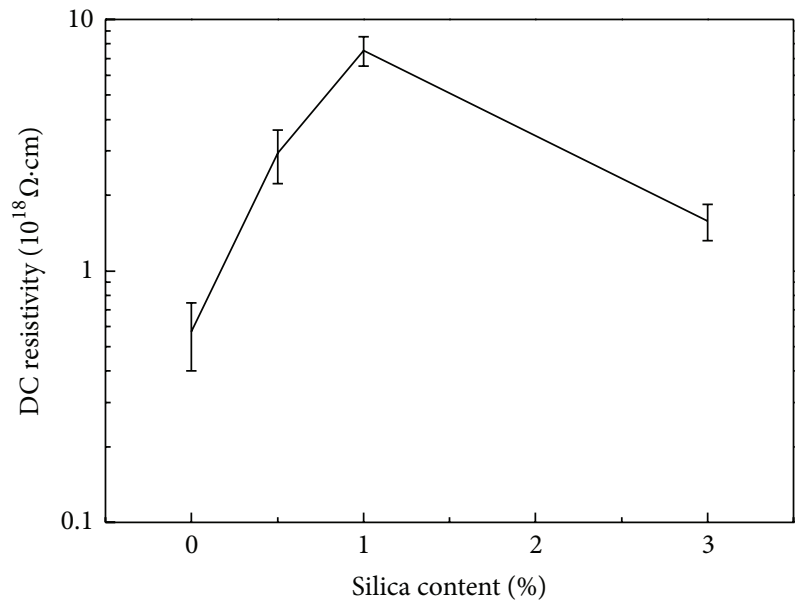

(b)

FIGURE 8: Leakage current density (a) and DC resistivity (b) of sPP and its nanocomposites subjected to an electric field of $6 \mathrm{kV} / \mathrm{mm}$ at room temperature.

TABLE 3: Comparison between sPP/chl-silica nanocomposites and XLPE.

\begin{tabular}{lcc}
\hline Properties & sPP/chl-silica nanocomposites & XLPE \\
\hline Operating temperature & $70^{\circ} \mathrm{C}$ & $70^{\circ} \mathrm{C}[11,12]$ \\
Breaking elongation & $\geq 200 \%$ & $\geq 200 \%[13]$ \\
Tensile yield strength $\left(25^{\circ} \mathrm{C}\right)$ & $\geq 10 \mathrm{MPa}$ & $22 \mathrm{MPa}[14]$ \\
DC volume resistivity & $\geq 10^{17} \Omega \cdot \mathrm{cm}(6 \mathrm{kV} / \mathrm{mm})$ & $0.92 \times 10^{17} \Omega \cdot \mathrm{cm}(10 \mathrm{kV} / \mathrm{mm})[15]$ \\
Space charge characteristics & No space charge accumulation in & Small amount of heterospace charge near anode \\
& $\mathrm{sPP}$ with $0.5 \mathrm{phr}$ and $1 \mathrm{phr}$ chl-silica & $(-50 \mathrm{kV} / \mathrm{mm})[15]$
\end{tabular}




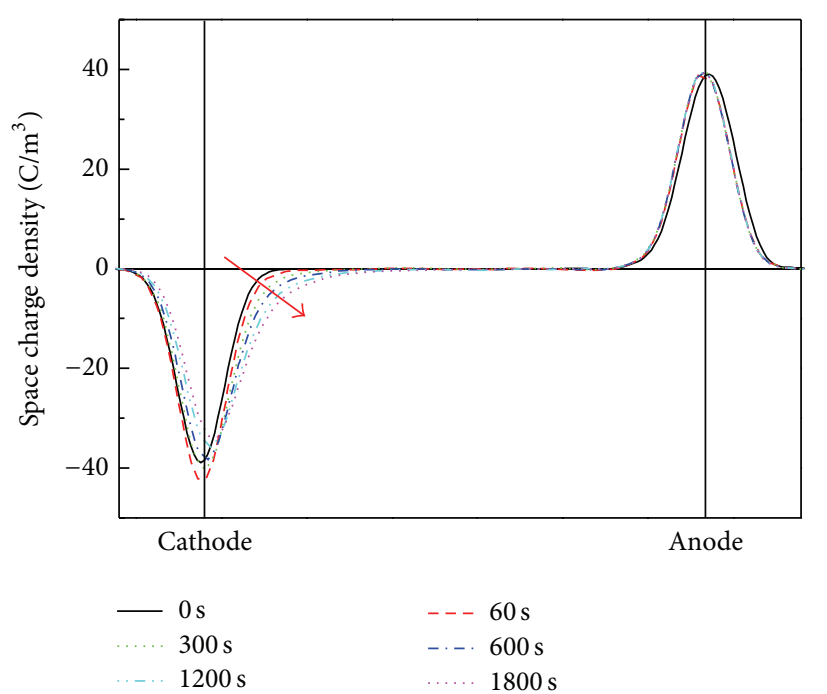

(a)

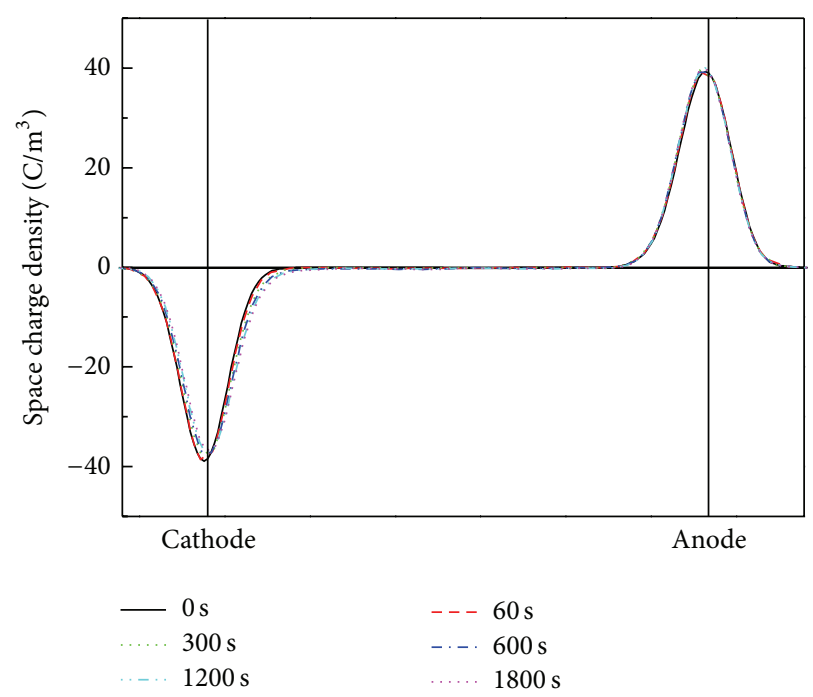

(c)

FIGURE 9: Space charge distribution during polarization in (a) sPP, $60 \mathrm{kV} / \mathrm{mm}$ for $1800 \mathrm{~s}$.

\section{Acknowledgment}

This work was supported in part by the National Basic Research Program of China (973 Project) under Grant 2014 CB239500.

\section{References}

[1] I. L. Hosier, A. S. Vaughan, and S. G. Swingler, "An investigation of the potential of ethylene vinyl acetate/polyethylene blends for use in recyclable high voltage cable insulation systems," Journal of Materials Science, vol. 45, no. 10, pp. 2747-2759, 2010.

[2] J. H. Kwon, M. H. Park, K. J. Lim, and H. K. Lee, "Investigation on electrical characteristics of HDPE mixed with EVA applied for recyclable power cable insulation," in Proceedings of the IEEE International Conference on Condition Monitoring and

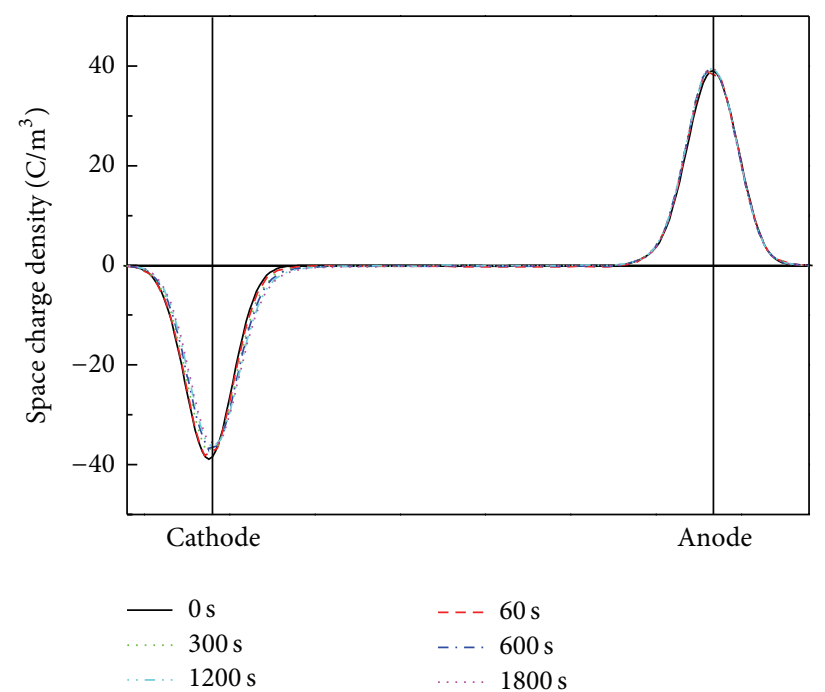

(b)

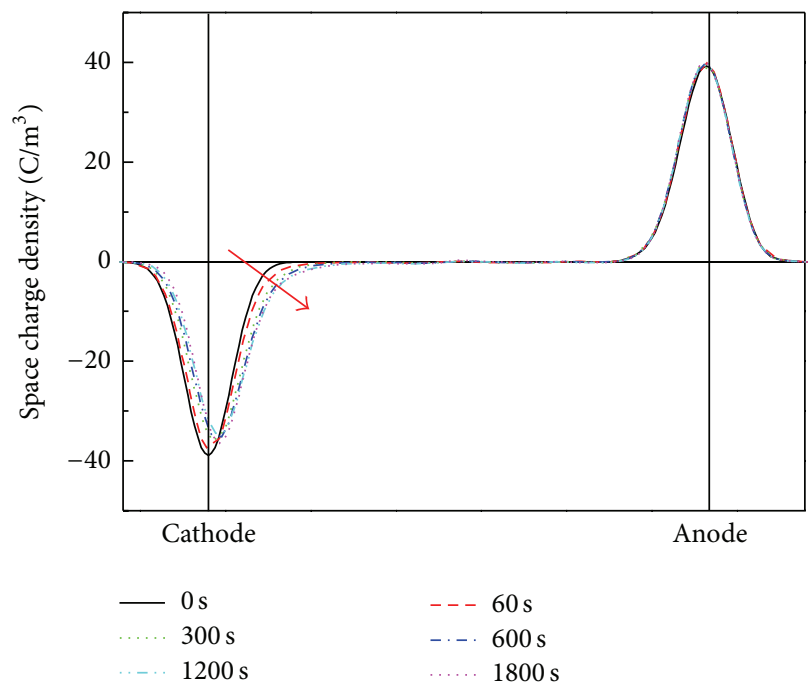

(d) 
the insulation of an electric power cable," Electrical Engineering in Japan, vol. 146, no. 1, pp. 18-26, 2004.

[7] D. W. Kim and K. Yoshino, "Morphological characteristics and electrical conduction in syndiotactic polypropylene," Journal of Physics D, vol. 33, no. 4, article 464, 2000.

[8] M. Roy, J. K. Nelson, R. K. MacCrone, and L. S. Schadler, "Candidate mechanisms controlling the electrical characteristics of silica/XLPE nanodielectrics," Journal of Materials Science, vol. 42, no. 11, pp. 3789-3799, 2007.

[9] C. W. Reed, "Functionalization of nanocomposite dielectrics," in Proceedings of the IEEE International Symposium on Electrical Insulation (ISEI '10), pp. 1-4, San Diego, Calif, USA, June 2010.

[10] L. T. Truong, Å. Larsen, B. Holme et al., "Dispersibility of silane-functionalized alumina nanoparticles in syndiotactic polypropylene," Surface and Interface Analysis, vol. 42, no. 6-7, pp. 1046-1049, 2010.

[11] C. P. Martin, The impact of mechanical stress on the mechanical integrity of XLPE cables [Ph.D. thesis], University of Southampton, Southampton, UK, 2004.

[12] I. L. Hosier, A. S. Vaughan, A. Campus, and U. Nilsson, "Morphology of novel XLPE cable systems," in Proceedings of the IEEE International Conference on Solid Dielectrics (ICSD '07), pp. 227-230, Winchester, UK, July 2007.

[13] G. C. Montanari, "Bringing an insulation to failure: the role of space charge," IEEE Transactions on Dielectrics and Electrical Insulation, vol. 18, no. 2, pp. 339-364, 2011.

[14] L. Boukezzi and A. Boubakeur, "Prediction of mechanical properties of XLPE cable insulation under thermal aging: neural network approach," IEEE Transactions on Dielectrics and Electrical Insulation, vol. 20, no. 6, pp. 2125-2134, 2013.

[15] L. Zhang, Y. Zhou, M. Huang, Y. Sha, J. Tian, and Q. Ye, "Effect of nanoparticle surface modification on charge transport characteristics in XLPE/SiO ${ }_{2}$ nanocomposites," IEEE Transactions on Dielectrics and Electrical Insulation, vol. 21, no. 2, pp. 424-433, 2014.

[16] P. Larkin, Infrared and Raman Spectroscopy; Principles and Spectral Interpretation, Elsevier, 2011.

[17] M. L. Cerrada, V. Rodríguez-Amor, and E. Pérez, "Effects of clay nanoparticles and electron irradiation in the crystallization rate of syndiotactic polypropylene," Journal of Polymer Science, Part B: Polymer Physics, vol. 45, no. 9, pp. 1068-1076, 2007.

[18] V. G. Gregoriou, G. Kandilioti, and S. T. Bollas, "Chain conformational transformations in syndiotactic polypropylene/layered silicate nanocomposites during mechanical elongation and thermal treatment," Polymer, vol. 46, no. 25, pp. 11340-11350, 2005.

[19] T. Tanaka, "Dielectric nanocomposites with insulating properties," IEEE Transactions on Dielectrics and Electrical Insulation, vol. 12, no. 5, pp. 914-928, 2005.

[20] Y. Cao and P. C. Irwin, "The electrical conduction in polyimide nanocomposites," in Proceedings of the Conference on Electrical Insulation and Dielectric Phenomena. Annual Report, pp. 116119, IEEE, October 2003.

[21] Y. Zhang, J. Lewiner, C. Alquié, and N. Hampton, "Evidence of strong correlation between space-charge buildup and breakdown in cable insulation," IEEE Transactions on Dielectrics and Electrical Insulation, vol. 3, no. 6, pp. 778-783, 1996. 

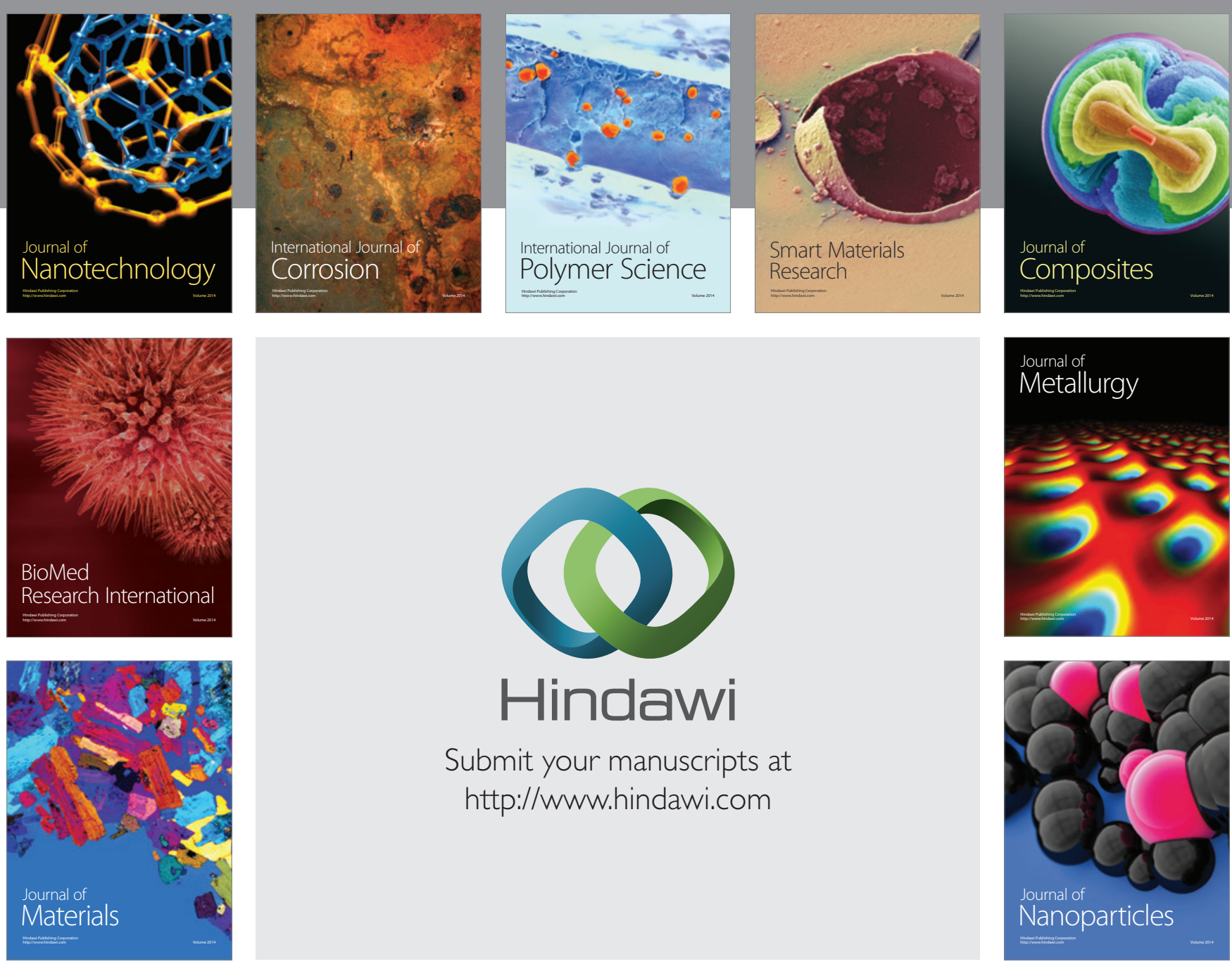

Submit your manuscripts at http://www.hindawi.com
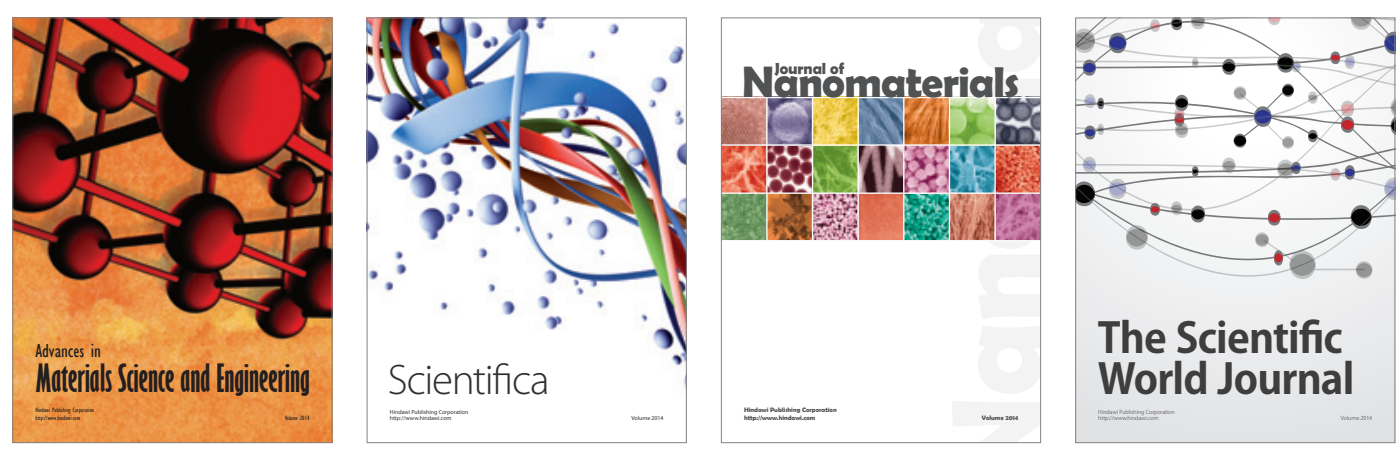

\section{The Scientific World Journal}
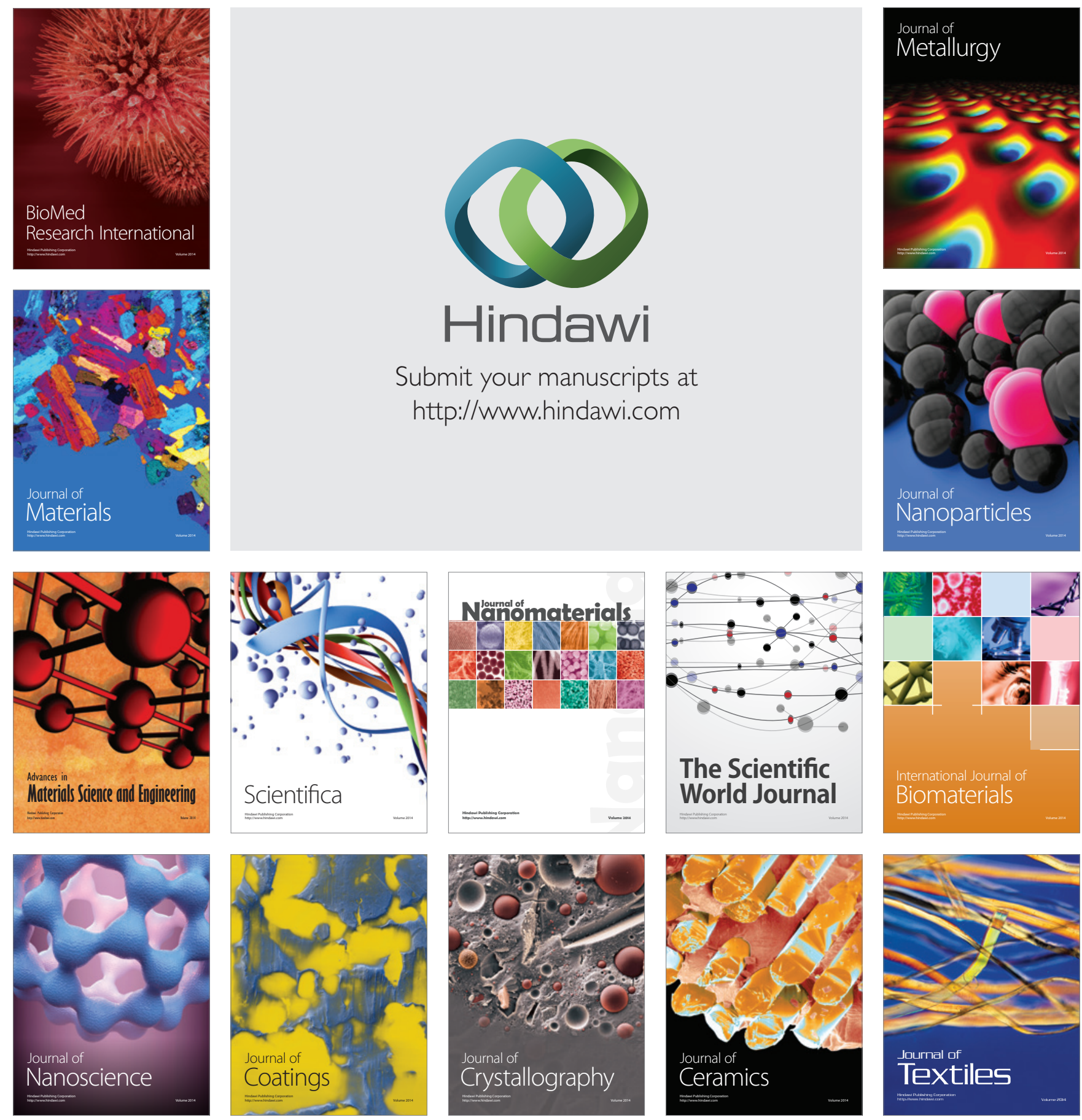